Figure 1. Primary cell of trospium triiodide

MS32 Polymorphs, cocrystals, solvates, salts: a jungle for scientists and industries
Keywords: Salt, Organic ions, X-ray diffraction, Structure, Crystallography,
Chairs: Catharine Esterhuysen, Martin Schmidt

\section{MS32-P1 Structural Characterization of Various Salts of Trospium: From Small Change of Anion to Huge Unit Cell}

\author{
Martin Babor ${ }^{1}$, Eliška Skořepováa ${ }^{1}, J^{2} \breve{C}$ ejka $^{1}$
}

1. Department of Solid State Chemistry, University of Chemistry and Technology Prague, Czech Republic

email: baborm@vscht.cz

Trospium chloride (TCl) is a drug used to treat urge incontinence and frequent urination. Four different salts of trospium are known. They are trospium chloride, bromide, iodide and saccharinate. A detailed structure characterization is available only for TCl. In this work, a single-crystal X-ray diffraction was used for structure characterization. The salts of trospium were prepared in two different ways. Inorganic salts were made from a mixture of $\mathrm{TCl}$ with hydrobromic or hydriodic acid in varied ratio. Organic salts were prepared from a mixture of $\mathrm{TCl}$ and sodium salt of organic acid in ratio 1:1. New structures of four forms were solved (trospium iodide, triiodide and two polymorphs of trospium saccharinate). New structures and structure of $\mathrm{TCl}$ were compared to each other and high similarity was found among them. There is some small trend between the anion shape and the structure, but different anions create similar structure as well. An extreme example of the structure dependent variation on the anion is trospium triiodide, which crystallized in a really huge unit cell $\left(\mathrm{V}=19438.7 \AA^{3}\right)$ almost seventy times larger than in the other structures of the trospium salts.

This work was supported by the Grant Agency of Czech Republic, Grant no. 106/16/10035S and received financial support from specific university research (MSMT No 20/2016).

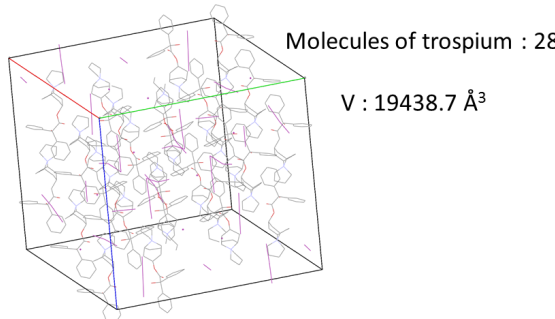

